\title{
A NOTE ON THE DIVISIBILITY OF CERTAIN CHERN NUMBERS
}

\author{
LEONIDAS CHARITOS AND STAVROS PAPASTAVRIDIS
}

\begin{abstract}
If $M$ is a weakly almost complex manifold, then $c_{r}(M) \in H^{24}(M ; Z)$ is the $r$ th Chern class of its normal bundle.

THEOREM 1. If $m, r$ are natural numbers with $r<m$, then there exists a $2 m$-fold $M_{0}$, compact, closed and weakly almost complex, so that the normal characteristic number $\left\langle c_{r}\left(M_{0}\right) c_{m-r}\left(M_{0}\right),\left[M_{0}\right]\right\rangle$ is a power of 2.
\end{abstract}

1. Introduction. First, let us set the terminology. In this note the word "manifold" will mean a closed, compact $C^{\infty}$ differentiable weakly almost compact manifold. All cohomology groups will have integer coefficients. If $M$ is a $2 m$-fold, then we define the normal characteristic number

$$
\Delta_{r}^{m}(M):=(-1)^{m}\left\langle c_{r}(M) c_{m-r}(M),[M]\right\rangle
$$

for $0 \leqslant r \leqslant m$ (the index $m$ will be suppressed from $\Delta_{r}^{m}(M)$, when it will be clear from the context).

In [4], E. Rees and E. Thomas determined the highest power of 2 which divides all the numbers $\Delta_{r}^{m}(M)$, where $M$ ranges over all $2 m$-folds. In this note we will prove that the greatest common divisor of these numbers is the power of 2 determined by Rees-Thomas. (Note. Actually Rees-Thomas proved this assertion for $r=0,1,2$.) The question of divisibility at Chern-numbers has an interest of its own (see [2], where some calculations are done for low dimensional cases). The proof splits naturally in two cases. By elaborate computations with products of complex projective spaces we prove that there are manifolds $M$ so that $\Delta_{r}^{m}(M)$ is not divided by 3 . The same assertion for the case of prime numbers greater than 3 is based on the work of E. Brown and F. Peterson [1].

2. Computations. Our next lemma puts together a few well-known facts about characteristic numbers.

LEMMA 2. (a) $\Delta_{r}^{m+n}(M N)=\sum_{i+j=r} \Delta_{i}^{m}(M) \Delta_{j}^{n}(N)$, where $M$ is a $2 m$-fold and $N$ is a $2 n$-fold.

(b) If $P_{n}$ is the $n$-complex projective space, then

$$
\Delta_{r}^{n}\left(P_{n}\right)=\left(\begin{array}{c}
n+r \\
n
\end{array}\right)\left(\begin{array}{c}
2 n-r \\
n
\end{array}\right)
$$

(The case $P_{0}=$ point is allowed.)

(c) If $P_{1}^{n}$ is the product $P_{1} \times P_{1} \times \cdots \times P_{1}$ (n times), then $\Delta_{r}^{n}\left(P_{1}^{n}\right)=\left(\begin{array}{c}n \\ r\end{array}\right) 2^{n}$.

Received by the editors December 15, 1980.

1980 Mathematics Subject Classification. Primary 57R20, 57R77.

Key words and phrases. Characteristic number, Hattori-Stong Theorem. 
Proof. See [4].

The next lemma is very well known.

LeMMA 3. Let $a=a_{0}+a_{1} p+\cdots+a_{n} p^{n}+\ldots, b=b_{0}+b_{1} p+\cdots+b_{n} p^{n}$ $+\ldots$ where $a, b$ are natural numbers, $p$ is a prime number and $a_{0}$, $a_{1}, \ldots, a_{n}, \ldots, b_{0}, b_{1}, \ldots, b_{n}, \ldots=0,1, \ldots,(p-1)$. Then

$$
\left(\begin{array}{l}
\alpha \\
\beta
\end{array}\right) \equiv\left(\begin{array}{l}
a_{0} \\
b_{0}
\end{array}\right)\left(\begin{array}{l}
a_{1} \\
b_{1}
\end{array}\right) \cdots\left(\begin{array}{l}
a_{n} \\
b_{n}
\end{array}\right) \ldots, \quad \bmod p .
$$

Corollary 4. (a)

$$
\Delta_{r}\left(P_{1}^{3^{k}}\right) \equiv \begin{cases}0 \bmod 3 & \text { if } 0<r<3^{k} \\ -1 \bmod 3 & \text { if } r=0,3^{k}\end{cases}
$$

(b)

$$
\Delta_{r}\left(P_{1}^{2} \cdot 3^{k}\right) \equiv \begin{cases}0 \bmod 3 & \text { if } r \neq 0,3^{\mu}, 2 \cdot 3^{k} \\ 1 \bmod 3 & \text { if } r=0,2 \cdot 3^{k} \\ -1 \bmod 3 & \text { if } r=3^{k}\end{cases}
$$

(c)

$$
\Delta_{r}\left(P_{3^{k}}\right) \equiv \begin{cases}1 \bmod 3 & \text { if } 0<r<3^{k} \\ -1 \bmod 3 & \text { if } r=0,3^{k}\end{cases}
$$

Proof. It comes from the combination of Lemma 2, parts (b) and (c), and Lemma 3.

LEMMA 5. Let $r, k, n$ be natural numbers so that $r<3^{k}<n-r$. Then $\Delta_{r}^{n}\left(M P_{1}^{3^{k}}\right)$ $\equiv-\Delta_{r}^{n-3^{k}}(M) \bmod 3$ for all $2\left(n-3^{k}\right)$-folds $M$.

Proof. Immediate from Lemma 2(a) and Corollary 4(a).

LEMMA 6. Let $r, k, n$ be natural numbers so that $r<3^{k}<n$ and $(n-r)<3^{k}$. Then $\Delta_{r}^{n}\left(P_{3^{k}} P_{1}^{n-3^{k}}\right) \not \equiv \bmod 3$.

Proof. From Lemma 2(a), (c) and Corollary 4(c), we have

$$
\begin{aligned}
\Delta_{r}^{n}\left(P_{3^{k}} P_{1}^{n-3^{k}}\right) & \equiv \pm \sum_{i=0}^{n-3^{k}}\left(\begin{array}{c}
n-3^{k} \\
i
\end{array}\right) \bmod 3 \\
& \equiv \pm(1+1)^{n-3^{k}} \bmod 3 \\
& \equiv \pm 1 \bmod 3 .
\end{aligned}
$$

LeMma 7. Let $r, n, k$ be natural numbers so that $3^{k} \leqslant n-r<2 \cdot 3^{k}<n$ and $2 \cdot 3^{k}<r$. Then

$$
\Delta_{r}^{n}\left(M \cdot P_{1}^{2 \cdot 3^{k}}\right) \equiv-\Delta_{r-3^{k}}^{n-2 \cdot 3^{k}}(M) \bmod 3
$$

for all $2 \cdot\left(n-2 \cdot 3^{k}\right)$-folds $M$.

Proof. It is an easy calculation from Lemma 2(a) and Corollary 2(b). 
LEMMA 8. Let $r, n, k$ be natural numbers so that $3^{k}-r<n<3^{k+1}$. Then $\Delta_{r}^{n}\left(P_{1}^{n}\right) \neq 0 \bmod 3$.

Proof. It is an immediate consequence of Lemma 2(c) and Lemma 3.

3. Proof of the main result. Now we are ready to give the

Proof of Theorem 1. It is enough to prove that if $p$ is a prime number greater than 2 , then there is a $2 m$-fold $M_{0}$ so that $\Delta_{r}^{m}\left(M_{0}\right) \neq 0 \bmod p$.

We distinguish two cases, depending on whether $p=3$ or $p$ is greater than 3 .

Case 1. Let $p=3$. Then the result follows by induction on $n$, using Lemmas $5,6,7,8$ combined with the obvious remark that we can interchange the roles of $r$ and $(m-r)$ because, clearly, $\Delta_{r}^{m}=\Delta_{m-r}^{m}$. If $m=0$ our assertion is obvious. In the other cases Lemma 5 and 7 permit us to reduce our assertion to a lower $m$, with two exceptions, which are covered by Lemmas 6 and 8 .

Case 2. Let $p>3$. Let $A$ be the subalgebra of the mod-p Steenrod algebra, generated by the reduced powers $P^{1}, P^{2}, \ldots . \mathrm{J}$. Milnor, in [3, Theorem 2], proved that $H^{*}\left(M U ; Z_{p}\right)$ is a free $A$-module, and a free basis is determined by the elements of the form

$$
s\left(a_{1}, a_{2}, \ldots, a_{k}\right) U=\left(\sum t_{1}^{a_{1}} t_{2}^{a_{2}} \ldots t_{k}^{a_{k}}\right) U,
$$

where none of the integers $a_{1}, a_{2}, \ldots, a_{k}$ is of the form $p^{i}-1$. It is easy to observe that if $c_{a}, c_{b} \in H^{*}\left(B U ; Z_{p}\right)$ are the mod-p Chern classes defined by $c_{a}=$ $\sum t_{1} \cdots t_{a}$ and $c_{b}=\sum t_{1} \cdots t_{b}$, then $c_{a} c_{b}=\left(\sum t_{1} \cdots t_{a}\right)\left(\sum t_{1} \cdots t_{b}\right)$, so $c_{a} c_{b} U$ is a sum of elements of Milnor's basis described above.

This is not true for $p=3$, because in this case the number 2 is of the form $\left(p^{i}-1\right)$.

On the other hand, Brown and Peterson, in [1, Theorem 1.2], gave criteria when a mod-p characteristic class $x \in H^{n}\left(B U ; Z_{p}\right)$ is zero on all $2 n$-folds (meaning as a normal characteristic number) and the criterion is the following: If $\left\{x_{i}\right\}_{i \in I}$ is an $A$-basis for $H^{*}\left(M U ; Z_{p}\right)$, then there must be a $a_{i} \in A$ for $i \in I$ with $\operatorname{deg} a_{i}>0$ so that $x U=\sum_{i \in I} a_{i} x_{i}$. Of course Brown and Peterson deal with tangential characteristic numbers, but their proof works the same (it is even simpler) with normal characteristic numbers.

From the remarks above, clearly $c_{a} c_{b} U$ cannot meet this criterion.

\section{REFERENCES}

1. E. Brown, Jr. and F. P. Peterson, Relations among characteristic classes. II, Ann. of Math. (2) 82 (1965), 356-363.

2. A. Liulevicius, On the birth and death of elements in complex cobordism (Proc. Conf. Homotopy Theory, Evanston, Illinois, 1974), Sociedad Matematica Mexicana, Mexico, 1975, pp. 78-98.

3. J. W. Milnor, On the cobordism ring $\Omega^{*}$ and a complex analogue, Amer. J. Math. 82 (1960), 505-521.

4. E. Rees and E. Thomas, On the divisibility of certain Chern numbers, Quart. J. Math. Oxford Ser. (2) 28 (1977), 389-401.

Chair of Algebra B, University of Patras, Patras, Greece 